\title{
Correction: Fabrication of Flexible, Highly Reproducible, and Hydrophobic Surface-enhanced Raman Scattering Substrates Through Silver- Nanoparticle Inkjet Printing
}

\author{
[J. Korean Phys. Soc. 76, 1025 (2020)] \\ DOI: $10.3938 / \mathrm{jkps} .76 .1025$ \\ BongJun KIM and Honggu CHUN* \\ Department of Biomedical Engineering, Korea University, Seoul 02841, Korea \\ Seong Jin BACK and Gyeong Bok JUNG ${ }^{\dagger}$ \\ Department of Physics Education, Chosun University, Gwangju 61452, Korea
}

DOI: $10.3938 /$ jkps. 76.1154

The first author's name should be changed from "BongJun KIM" to "Bongjoon KIM". 